\title{
Structure interne du glacier rocheux actif de Las Argualas (Pyrénées aragonaises, Espagne)
}

\author{
D. Fabre (1), F. Garcia (2), M. Evin (3), R. Martinez (2), E. Serrano (4), A. Assier (1), C. Smiraglia (5)
}

(1) Université Joseph Fourier, Grenoble, France

(2) Université Polytechnique de Valence, Espagne

(3) Université Jules Verne, Amiens, France

(4) Université de Cantabrique, Santander, Espagne

(5) Université de Milan, Italie

Les glaciers rocheux sont constitués d'un mélange de glace et de débris formés au pied de parois rocheuses, à la limite des neiges permanentes. La glace provient de la transformation de la neige des avalanches, du regel des eaux de fonte des névés ou, quelquefois, de lentilles héritées d'un ancien glacier. A cause du fluage de la glace, le matériau constituant le glacier rocheux actif (qu'on pourrait qualifier de «béton de glace ") est susceptible de se mouvoir sur les pentes et de donner des formes de langues, de bourrelets et de sillons. Dans certains cas, un mouvement actuel a pu être mesuré. Dans d'autres, la glace constituant le liant des blocs a entièrement fondu et on n'observe plus que des formes héritées.

Pour mieux connaître les glaciers rocheux et leur mécanisme, il importe de pouvoir déterminer clairement la présence ou l'absence de glace, de mesurer la profondeur et l'épaisseur des niveaux qui en contiennent et, si possible, de faire une hypothèse sur leur nature et leur origine.

En l'absence de sondage mécanique, délicat et très onéreux dans ce contexte difficile, la prospection électrique peut répondre à ces questions, au moins de manière semiquantitative. En effet, on peut espérer un très bon contraste de résistivité entre les éboulis, le rocher et la glace. La résistivité de cette dernière est très élevée (100 à 1000 fois plus grande que celle des éboulis) et même lorsque la glace n'occupe qu'une partie du volume total (cas du béton de glace) sa présence doit se faire sentir. D'autre part, la résistivité des débris grossiers (cas fréquent dans le contexte des glaciers rocheux) peut être 2 à 5 fois supérieure à celle du rocher en place.

La méthode électrique a été appliquée par plusieurs d'entre nous avec succès sur différents glaciers rocheux depuis 1986, particulièrement dans les Alpes [1,2]. Le présent article en montre l'application à un site des Pyrénées espagnoles, sur un glacier rocheux actif pour lequel des études géomorphologiques [3] et topographiques sont engagées.

\section{LE SITE ÉTUDIÉ}

Le secteur d'étude se trouve dans le massif des Picos del Infierno, à l'Est du village de Sallent de Gallego et non loin du massif granitique frontalier du Balaïtous (voir fig. 1).

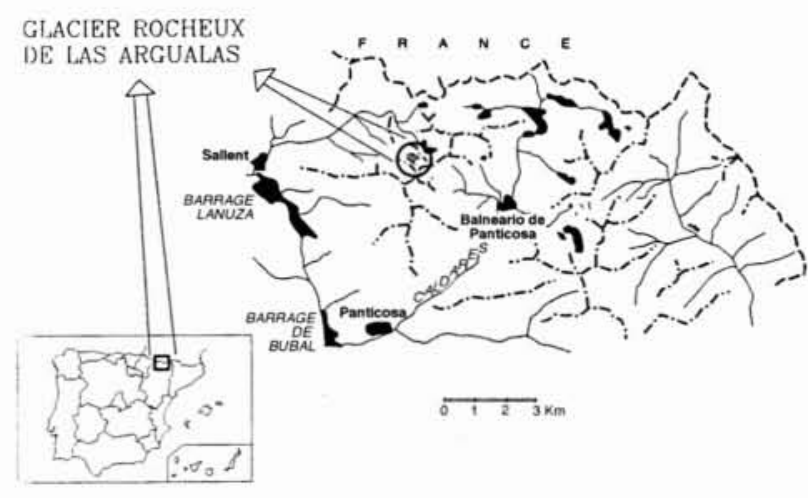

1. Situation générale du secteur étudié.

Le substratum géologique est constitué de terrains appartenant à l'auréole métamorphique du batholite de Panticosa. Il s'agit principalement de schistes et calcaires métamorphiques.

Les mesures sont concentrées sur le glacier rocheux de Las Argualas, superbe forme de $3 \mathrm{~km}^{2}$ ( $400 \mathrm{~m}$ de large sur $750 \mathrm{~m}$ de long environ), située dans un cirque d'altitude entre 2520 et $2750 \mathrm{~m}$ d'altitude (orientation W-NW). La crête dominant le glacier rocheux culmine à $3060 \mathrm{~m}$ au pic d'Argualas.

La zone a fait l'objet d'une étude géomorphologique très complète [4] et de mesures topographiques durant les étés 1991, 1993 et 1994. Celles-ci montrent un mouvement sensible du glacier rocheux, variable selon les points (fig. 2), mais en moyenne compris entre 20 et $40 \mathrm{~cm} / \mathrm{an}$ dans la partie centrale du glacier rocheux.

La pente du glacier rocheux est globalement de $25^{\circ}$ vers le NW avec, comme cela est classique pour les glaciers rocheux, un secteur amont de pente moindre $\left(13\right.$ à $\left.19^{\circ}\right)$ et un front raide $\left(42^{\circ}\right)$ à l'aval.

D'après l'altitude et des considérations climatiques [4], l'hypothèse la plus vraisemblable serait que la forme encore en mouvement aujourd'hui aurait été constituée ou réactivée au Petit-Age-de-Glace et ne serait plus en équilibre avec les conditions climatiques actuelles.

Les mouvements encore actuels, la température des eaux de sources situées à l'aval faisaient penser à la présence 


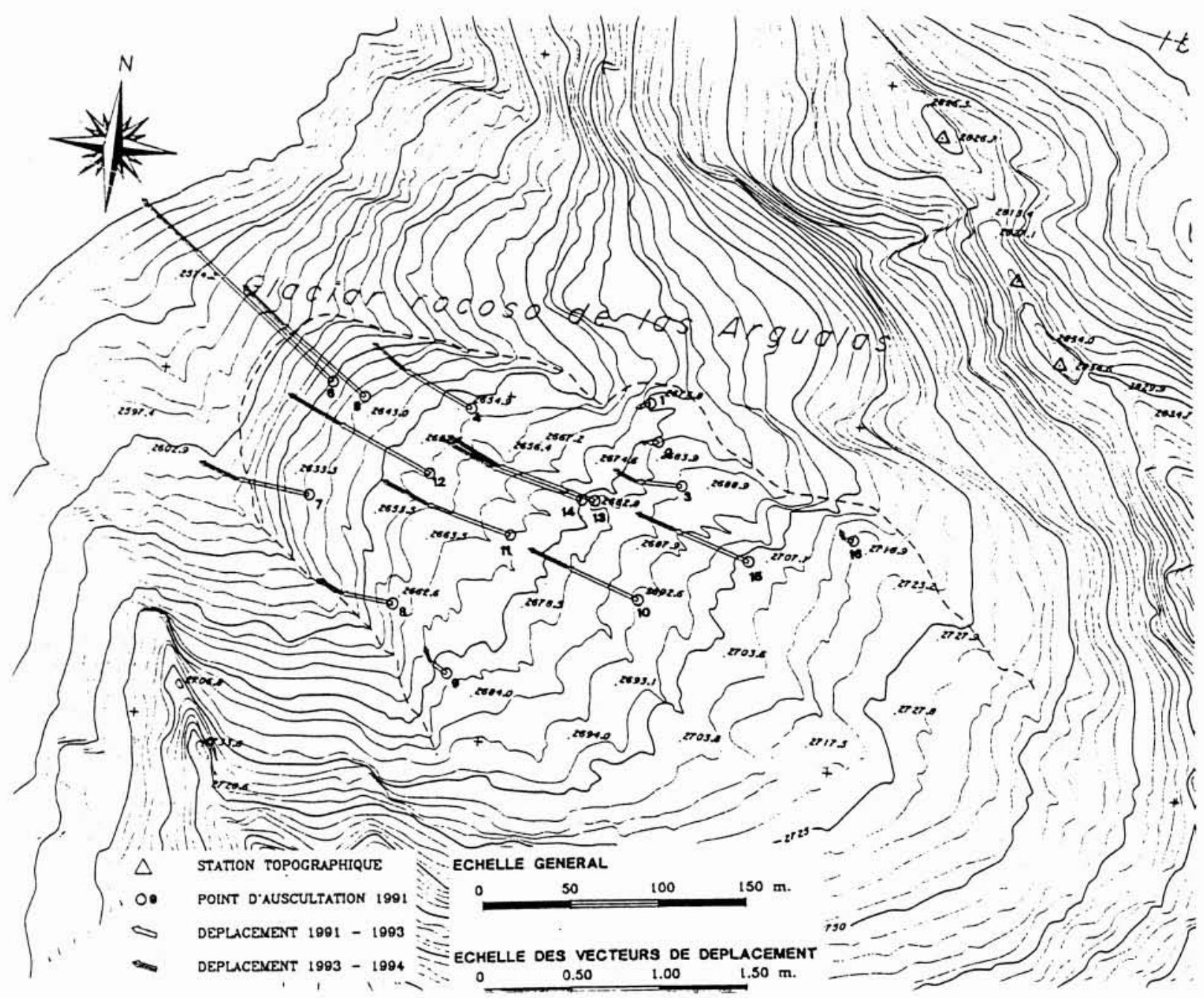

2. Les mouvements mesurés sur le glacier rocheux de Las Argualas entre 1991 et 1994.

de glace dans les sédiments. Il appartenait à la prospection électrique réalisée de confirmer cette hypothèse et de préciser la profondeur, l'épaisseur et la nature des niveaux englacés.

\section{II — LA PROSPECTION GÉOÉLECTRIQUE RÉALISÉE}

Elle a consisté en une série de sondages électriques par la méthode classique Schlumberger, à l'aide d'un appareil MAATEL BMI, spécialement adapté pour la mesure des fortes résistivités [5].

\subsection{Sondages d'étalonnage}

Deux sondages ont été réalisés en dehors du glacier rocheux actif dans le but d'étalonner les résistivités de deux niveaux géologiques bien caractérisés :
- le substrat (secteur de Salvas, altitude 2200 , sondage AR 8) ;

- le glacier rocheux fossile de Salvas, constitué a priori d'éboulis sans glace (altitude $2240 \mathrm{~m}$ : sondage AR 7).

Les courbes de résistivité pour ces deux sondages sont données sur la figure 3.

La résistivité du substrat est trouvée voisine de $2500 \Omega \mathrm{m}$. Il faut noter que la partie superficielle donne une résistivité plus forte due à la zone d'altération où des fissures peuvent être ouvertes. Celle-ci ne paraît pas dépasser $2 \mathrm{~m}$ d'épaisseur.

Le glacier rocheux ancien donne une courbe typique de forme sans glace. On y distingue trois couches :

- couche superficielle de débris un peu plus conducteurs (humidité, un peu de terre végétale) $p_{1}=9000 \Omega \mathrm{m}$, $h_{1}=0,8 \mathrm{~m}$;

- couche intermédiaire de débris sans fines, $p_{2}=22000 \Omega \mathrm{m}, h_{2}$ de 6 à $8 \mathrm{~m}$ d'épaisseur ;

- couche profonde où l'on trouve la résistivité du substrat $(2500 \Omega \mathrm{m})$. 


\section{pa ( $\Omega . m)$}

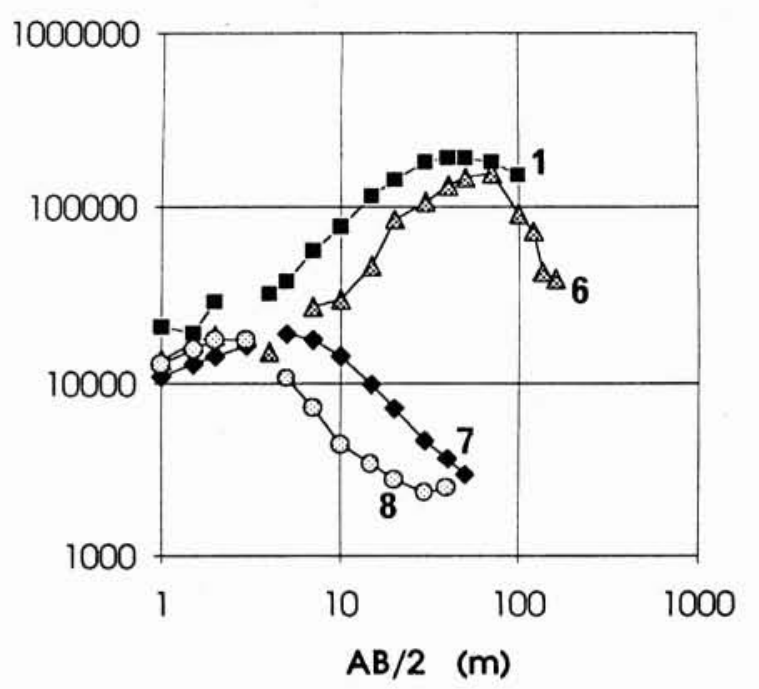

3. Courbes de sondages électriques sur le secteur de Salvas $\left(n^{\circ} 7\right.$ et $\left.n^{\circ} 8\right)$ et sur le glacier rocheux actif de Las Argualas ( $n^{\circ} 1$ et $\left.n^{\circ} 6\right)$.
2.2 Prospection sur le glacier rocheux actif d'Argualas

La surface relativement importante du glacier rocheux a permis la réalisation de 6 sondages relativement longs $(\mathrm{AB} / 2$ compris entre 70 et $150 \mathrm{~m}$ voir, figure 4$)$ :

- 2 longitudinaux : 511 dans la partie haute, plutôt en rive droite ; 566 , le plus long, dans la partie centrale ;

- 4 transversaux de haut en bas: 52 2, 533,544 et 555 .

Toutes les courbes de résistivité (voir figs. 3 et 5 ) sont caractéristiques d'une forme contenant de la glace : courbes globalement tricouches avec résistivité élevée de la deuxième couche. Dans le détail, ces sondages présentent des nuances qui vont être précisées plus loin. On attire l'attention sur l'importance de la résistivité de la couche englacée qui est une caractéristique de la richesse en glace du sédiment, comme toutes nos études antérieures l'ont montré [2].

\subsection{Première interprétation}

Les sondages transversaux dont trois courbes sont données sur la figure 5 font apparaître un niveau de sédiments

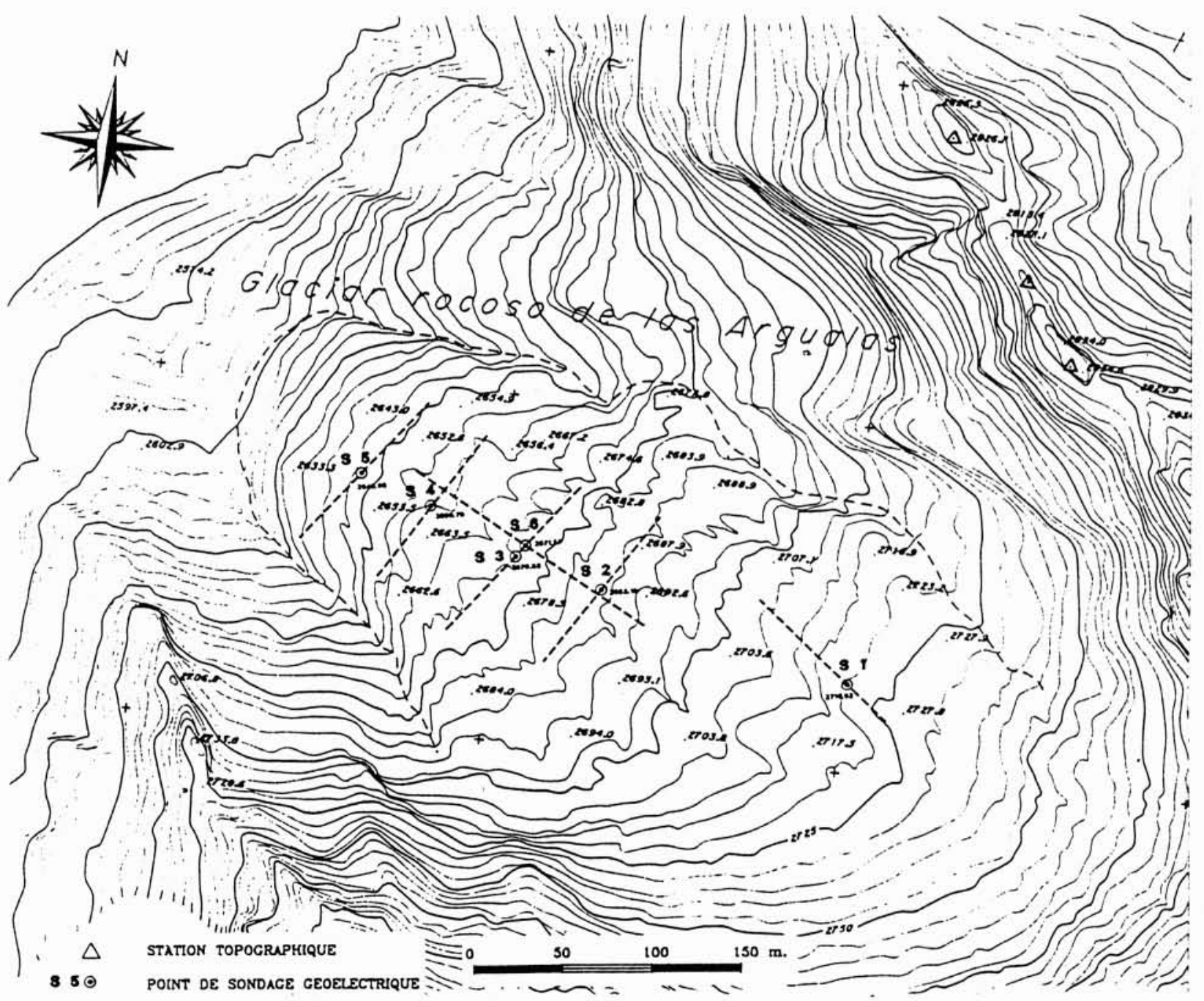

4. Implantation des sondages électriques sur le glacier rocheux de Las Argualas. 


\section{pa ( $(\Omega . m)$}

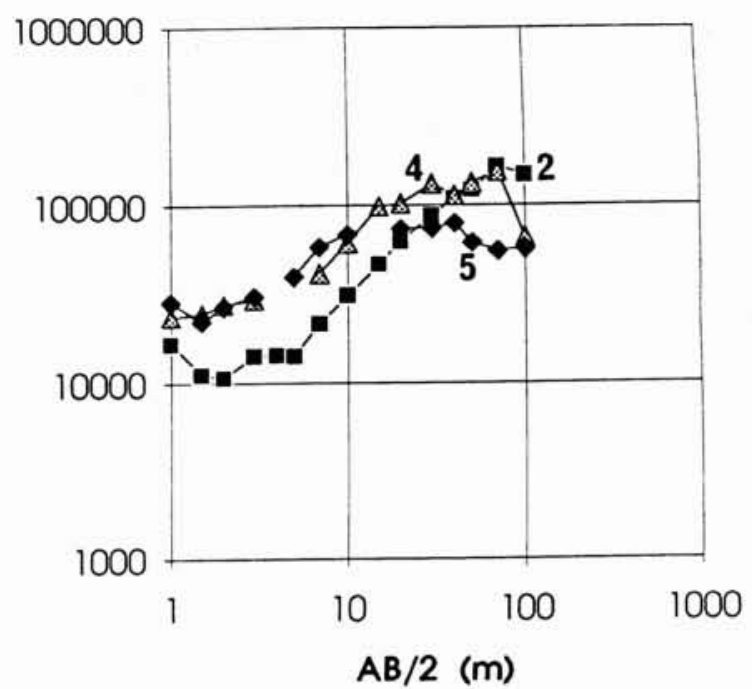

5. Courbes de sondages électriques transversaux sur le glacier rocheux actif de Las Argualas $\left(n^{\circ} 2, n^{\circ} 4\right.$ et $\mathbf{n}^{\circ} \mathbf{5}$ ).

englacés de résistivité comprise entre 200000 et $600000 \Omega \mathrm{m}$ (plus élevée à l'amont). Ceci fait penser à des sédiments riches en glace s'appauvrissant un peu d'amont vers l'aval. L'épaisseur de ce niveau englacé est compris entre 10 et 20 mètres, sa profondeur par rapport à la surface est de 2 à 4 mètres.

Le sondage AR 5, non loin du front du glacier rocheux donne la résistivité $\rho_{2}$ la plus faible $(200000 \Omega \mathrm{m})$, ce qui correspond à un sédiment moyennement riche en glace avec une épaisseur faible $(5 \mathrm{~m})$. Il surmonte un autre niveau de résistivité $45000 \Omega \mathrm{m}$ que l'on pourrait interpréter comme un niveau faiblement englacé. Ceci pourrait indiquer que l'appauvrissement en glace par fonte aurait lieu davantage par la base que par le haut.

Les sondages longitudinaux (fig. 3) confirment la présence d'une couche assez riche en glace dans la partie haute et au centre de l'accumulation $(800000 \Omega \mathrm{m})$. La différence de résistivité peut être liée à une forme un peu lenticulaire de la couche englacée, comme le suggèrent aussi des pentes de courbes localement proches de la pente maximale des abaques (AR 6 par exemple).

Le sondage AR 1, le plus haut, ne permet pas d'identifier clairement le substrat en dessous de la couche englacée. II en est de même, d'ailleurs, pour le sondage transversal le plus haut (AR 2). Ceci pourrait être expliqué par la présence d'une couche intermédiaire (sédiments englacés ou non). Cependant, à cause du trop petit nombre de mesures montrant la décroissance de la courbe de résistivité, il est difficile de conclure nettement sur ce point.

\section{CONCLUSIONS}

Les mesures topographiques réalisées montrent clairement le mouvement encore actuel du glacier rocheux de Las Argualas. La valeur moyenne de la vitesse $(25 \mathrm{~cm} / \mathrm{an})$ est comparable à certains résultats obtenus sur d'autres sites dans les Alpes du Sud [6,7]. Elle est le signe d'une nette activité.

Les sondages électriques attestent de la présence de glace dans l'accumulation détritique constituant le glacier rocheux. La résistivité mesurée nous permet d'affirmer qu'il s'agit de sédiments riches en glace avec une épaisseur de 10 à $20 \mathrm{~m}$ sous 2 à 4 mètres de débris sans glace. La richesse en gláce va en décroissant du haut en bas de la forme, surtout au niveau du dernier sondage près du front du glacier rocheux. Ceci confirme que les processus de fonte sont actuellement prédominants et que l'origine de la glace remonterait au moins au Petit-Age-de-Glace.

Les données acquises permettent maintenant de se faire une bonne idée sur la structure interne du glacier rocheux de Las Argualas et d'envisager une modélisation rhéologique de ses mouvements.

\section{Références}

[1] EVIN M.. FABRE D. (1990). - The distribution of permafrost in rock glaciers of the Southern Alps (France). Geomorphology, 3, pp. 51-71.

[2] ASSIER A., EVIN M., FABRE D. (1994). - Les relations glacier-glacier rocheux dans le haut vallon d'Asti (Queyras). C.R. Colloque S.H.F., Section de Glaciologie, Grenoble 1011 mars $94,8 \mathrm{p}$.

[3] Serrano E., Rubio V. (1989). - El glaciar rocoso activo de las Argualas (Pirineo Aragonés). Eria, $\mathrm{n}^{\circ}$ 19-20, pp. 195 198.

[4] Serrano E. (1991). - Geomorfologia glaciar de las montanas y valles de Panticosa y la Ribera de Biescas (Pirineo aragonés). Tesis doctoral, Universidad Autonoma de Madrid, $940 \mathrm{p}$.

[5] FABre D., Evin M. (1990), - Prospection électrique des milieux à très forte résistivité : le cas du pergélisol alpin. Proc. 6th Int. I.A.E.G. Congress, Amsterdam, Balkema ed. pp. 927-934.

[6] EVIN M., AsSIER A. (1983). - Mise en évidence de mouvements sur le glacier rocheux de Sainte-Anne (Queyras. Alpes du Sud, France). Revue de Géographie Alpine, LXXI $\mathrm{n}^{\circ} 2$, pp. 165-178.

[7] Evin M. (1987). - Structure, répartition et age des glacier: rocheux des Alpes du Sud. Thèse doct. état, Université de Grenoble 1, 309 p. 